\title{
Comparison of Green Communications Technology Oriented to WBAN
}

\author{
Tan Dou ${ }^{1, a}$, Liu Yi, ${ }^{1,2, b}$, Zhang Tian ${ }^{3, b}$ \\ ${ }^{1}$ Beijing Institute of Fashion Technology, Chaoyang District, Beijing, China \\ ${ }^{2}$ Beijing University of Post and Telecommunications, Beijing, China \\ ${ }^{3}$ Huadian(Beijing) Co-generation Co.LTD, Beijing, China \\ a metandou@163.com, bmableliuyi@163.com
}

Keywords: WBAN; Green Communications; ZigBee; NFC, Bluetooth

\begin{abstract}
Wireless Body Area Network is a branch of Wireless Sensor Network. Generally, it is apply to the aspect of telemedicine and health care, etc. Green communication which is a communication concept of sustainable development is the inevitable trend of the development of the communications industry, today. This article introduces and compared three kinds of green communication technology including WiFi, Bluetooth adn ZigBee, which are all suitable for Wireless Body Area Network(WBAN).
\end{abstract}

\section{Introduction}

Wireless Body Area Network(WBAN),is centered on the human Body, and composed of a variety of network elements related to human body, included personal terminal, Networking equipment, and communications network which is made up of sensors that distributed on or inside the human body, and around the human Body ${ }^{[1]}$. It can communicate with any network terminal ${ }^{[2]}$. It is not only a cross of multiple discipline(as shown in figure 1), but also has important practical significance and the industrialization prospects.

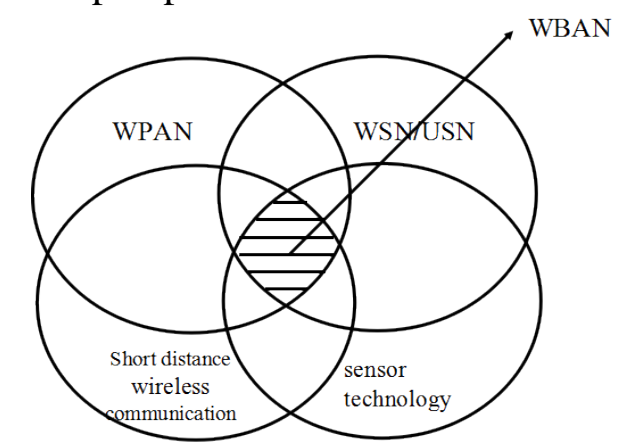

Fig.1.WBAN a cross of multiple discipline

\section{Architecture of WBAN}

a complete BAN should contains three layers(showed in Figure 2), the first layer consists of multiple sensor nodes, including EEG sensor, EMG sensor, ECG sensor, etc. These play a role not only in collecting the physiological parameters, but also processing information simply, and transfer the data to the sink node ${ }^{[3]}$. The second layer is the personal sever that people worn or family owned ${ }^{[4]}$. It received the data which from the sink node, and temporarily store these data after analysis and arrangement, then transfer the data with cable or wireless network to background database and store them. The third layer provided various application services, patients or their family members and doctors can access the database through the internet to know the medical information, and it also can remind medical staff to treat the patients duly.

\section{Green Communications}

Green communications is a new communication concept, aimed at achieve the goal about sustainable development by energy conservation, emissions reduction, reducing environmental pollution. It mainly adopted innovation technology, for instance, effective power amplifier, 
multi-carrier, distributed, intelligent temperature control. Green communications can reform the base station with the flexible site scene model, and finally reduce energy consumption.

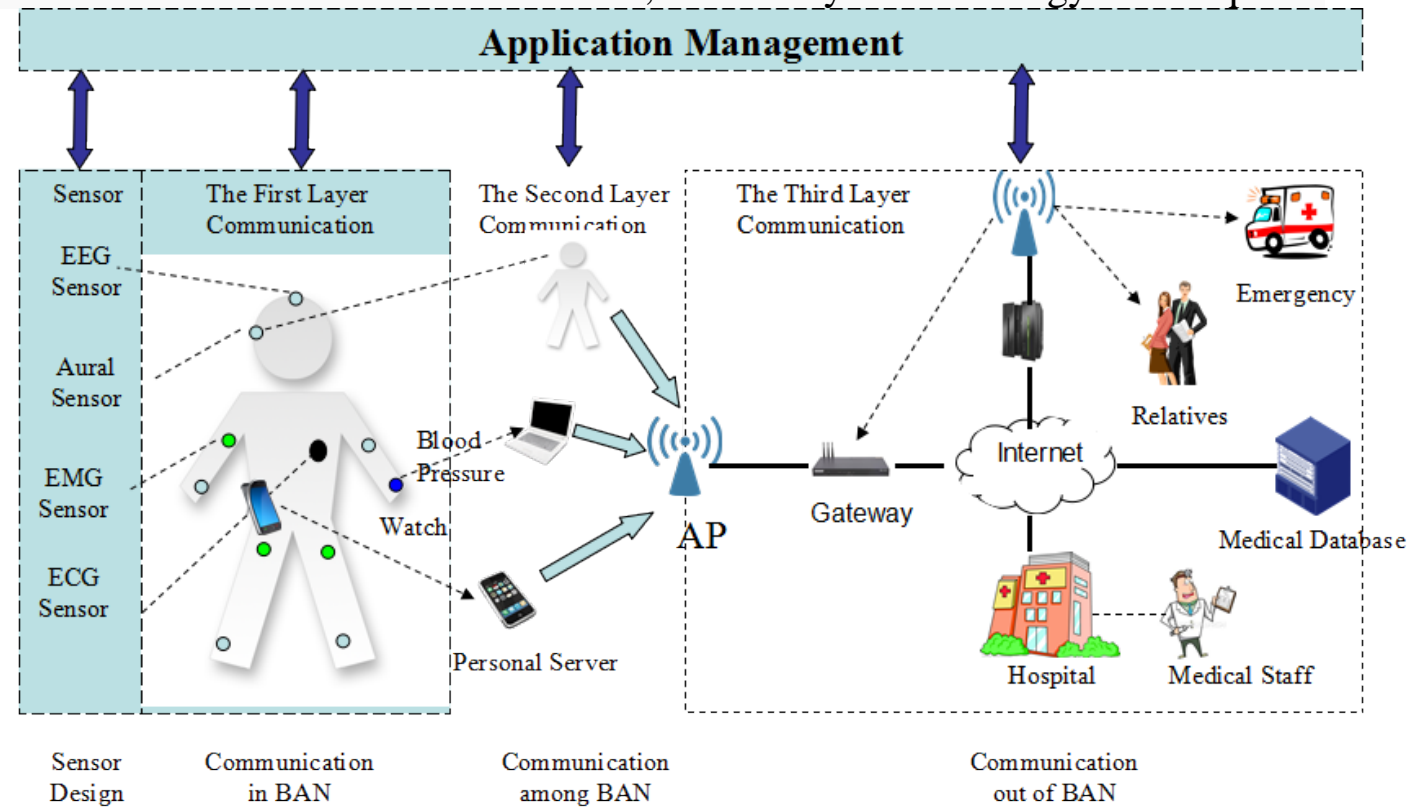

Fig.2 the architecture of WBAN

\section{Comparison of Green Communications Technology}

\section{I.Introduction of Green Communications Technology}

There're some technologies which are called Wifi,Bluetooth and Zigbee, and they're all common wireless communication technologies in the WBAN.They're relatively low power consumption, in line with the basic requirements of green communications. The following is the brief introduction of these three technologies.

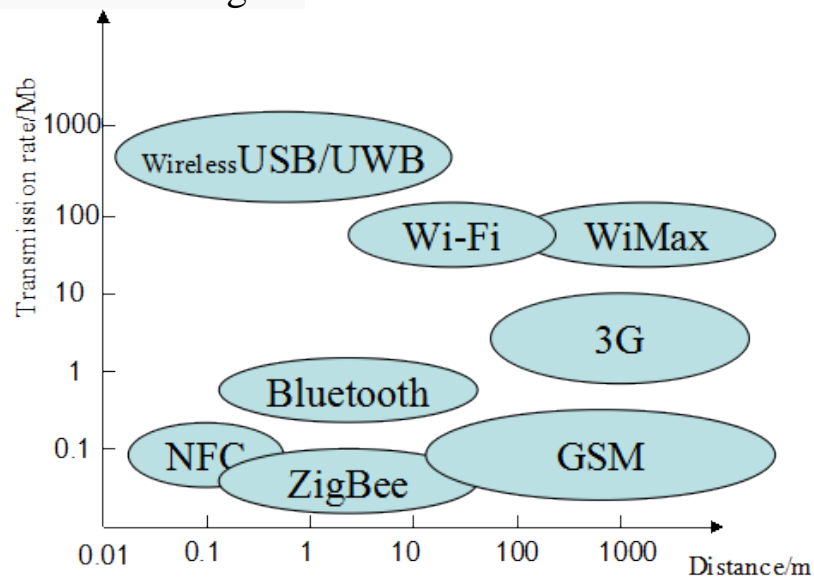

Fig.3.The Transmission coverage and transmission rate of several common Wireless communication technology

WiFi,also known as Wireless Fidelity, is the most widely used as a wireless network transmission technology today. It is a technology which can connect personal computer, hand-held devices and other terminals to each other by wireless.Bluetooth is a radio technology supporting the short distance communication equipment.It enables exchange of wireless information between devices such as mobile phones, PDA, wireless headsets, laptops, related peripherals, etc ${ }^{[5]}$. Due to the new low-power Bluetooth with the characteristics of ultra-low peak, average and idle mode power consumption, strong wireless coverage, fully backward compatible, its features make Bluetooth technology to become a good green communications technology.ZigBee, is a short-range, low-comlexity, low-data-rate two-way wireless communication technology ${ }^{[6]}$.It's mainly suitable for use in the field of automatic control and remote control, in brief, ZigBee also has the trait about low-cost, low-power consumption. 


\section{II.Comparison of Green Communications Technology}

There are many wireless communication technology in this area,such as,Bluetooth, WiFi, ZigBee, Near Field Communication(NFC), etc.Figure 3 shown the transmission coverage and transmission rate of several common wireless communication technology ${ }^{[7]}$.This article only talk about WiFi, ZigBee, Bluetooth, this three kinds technology.

Both of ZigBee and Bluetooth standards belong to wireless personal area network (WPAN), while WiFi belongs to WLAN technology standard.The Table1 below is the comparison of ZigBee, Bluetooth and $\mathrm{WiFi}^{[8]}$. The transmission energy of ZigBee is lower than the Bluetooth, however, the Bluetooth can be used for a limited amount of energy of low data transmission. The transmission rate of WiFi is much higher and more flexible than others. ZigBee is more reliable, low-power and cheaper. Bluetooth is cheap and convenient.

Table 1The comparison of ZigBee, Bluetooth and WiFi

\begin{tabular}{|c|c|c|c|c|c|c|c|}
\hline Function & ZigBee & Bluetooth & Wifi & Function & ZigBee & $\begin{array}{c}\text { Bluetoo } \\
\text { th }\end{array}$ & Wifi \\
\hline $\begin{array}{c}\text { IEEE } \\
\text { standard }\end{array}$ & 802.15 .4 & 802.15 .1 & 802.11g & Costs & low & medium & medium \\
\hline Bandwidth & $\begin{array}{c}2.4 \mathrm{GHz} \\
868 / 915 \\
\mathrm{MHz}\end{array}$ & $2.4 \mathrm{GHz}$ & $2.4 \mathrm{GHz}$ & $\begin{array}{c}\text { Modulation } \\
\text { Type }\end{array}$ & $\begin{array}{c}\text { BPSK } \\
\text { O-QPSK }\end{array}$ & GFSK & $\begin{array}{l}\text { BPSK } \\
\text { QPSK }\end{array}$ \\
\hline $\begin{array}{c}\text { Send } \\
\text { energy }\end{array}$ & $-25 \sim 0 \mathrm{~dB}$ & $0 \sim 10 \mathrm{~dB}$ & $18 \sim 27 \mathrm{~dB}$ & $\begin{array}{c}\text { Transmission } \\
\text { distance }\end{array}$ & $\begin{array}{c}10 \sim 100 \\
\mathrm{~m}\end{array}$ & $30 \mathrm{~m}$ & $10 \sim 300 \mathrm{~m}$ \\
\hline $\begin{array}{c}\text { The } \\
\text { maximum } \\
\text { transmissi } \\
\text { on rate }\end{array}$ & 250kbps & $1 \mathrm{Mpbs}$ & 54Mpbs & $\begin{array}{c}\text { The } \\
\text { maximum } \\
\text { number of } \\
\text { nodes }\end{array}$ & 65536 & 7 & 2007 \\
\hline $\begin{array}{c}\text { Spread } \\
\text { Spectrum }\end{array}$ & DSSS & FHSS & OFDM & $\begin{array}{l}\text { The number } \\
\text { of channels }\end{array}$ & 27 & 40 & 14 \\
\hline Security & AES & AES & AES & & & & \\
\hline
\end{tabular}

Comparing to Bluetooth, the protocol of ZigBee is simpler. ZigBee supports more sensor nodes, and needs relatively few hardware and software resources. While the cost of development of Bluetooth is higher, and Bluetooth needs large power consumption. Comparing to ZigBee, WiFi has higher cost at application,the larger power consumption, lower safety performance, and numerous resource requirement ${ }^{[9,10]}$. Only ZigBee has good advantages in the power consumption and cost. In theory, the protocol of ZigBee is more suitable for the data transmission of sign detection in WBAN $^{[11]}$. And compared to the other two techniques, ZigBee is more suitable at green communication.

\section{Conclusion}

By comparing three techniques on various characteristics, ZigBee is low-power, low-cost and a good green for WBAN communication technologies.Although the orther two don't have enough advantages in energy conservation and emission reduction, but it still have important applications in WBAN due to the other advantages. Three technologies are in constant development. In the future, these three technologies are likely to be combined with the advantages of each other to provide customers with the better and more comprehensive programs, and finally become a interdependent relationships.

\section{Acknowledgements}

This work was financially supported by Beijing College Student Research Training Program (2014, 2015), Open Project of Digital and Interactive Media Key Laboratory (KF2013-01, KF2013-13), Beijing Institute of Fashion Technology Teaching reform and innovation team project(JGTD-1404), PHR(IHLB)-Innovative Research Team PTTBIFT_TD_002, Beijing education reform project(2013-ms145). 


\section{References}

[1] KARAOGUZ J. Hish rate wireless personal area networks[J]. IEEE Comm.Magazine,2001, 9(12):96-1102.

[2] Yin'e Zhang, Xiaoqiang Chen.The key techniques and development of WBAN[J].TV Technology, 2004,34(4):70-72.

[3] Hanson M.A,Powell H.C,Barth A.T,et al.Body area sensor networks:challenges and opportunities[J].Computer,2009,42 (1):58-65.

[4] DeVaul R W,Schwartz S J,Pentland A,et al.MIThril: context-aware computing for daily life [Z].Whitepaper,MIT Media Lab,2001.

[5] Yue Yang,Chao Liu,Yongtai Chen.The Bluetooth technology and principle[J].Telecom Express, 2002,(2):17-20.

[6] Yue Fan, Ru Li, Jiyi Wang. Design and Realization of Bluetooth/ZigBee wireless network transmission system[J]Computer technology and development,2013,23(1):209-213.

[7] Yuehua Wang, Quan Yu..Analysis of the ZigBee technology[J].TV technology,2004(6):33-35.

[8] NFC Forum.NFC digital protocol technical specification 1.0[S].2010.

[9] Ting Sun,Yongtian Yang,Lihong Li.Current situation of the development of wireless sensor network technology[J].Electronic technology applications,2005(6):1-5.

[10] Jianzhong Li, Jinbao Li, Shengfei Shi. Sensor network and the concept of data management Concepts,issues and advance of sensor networks and date management of sensor networks[J]. Journal of software,2003(10):1717-1727.

[11] CHEN Min, GONZALEZ Sergio, VASILAKOS, Athanasios, et al. Body area networks: a survey[J]. Mobile Networks and Applications,2011(16):171-193. 